\title{
A Parallel Robot for Ankle Rehabilitation-Evaluation and its Design Specifications.
}

\author{
Christos E. Syrseloudis and Ioannis Z. Emiris
}

\begin{abstract}
This paper investigates several robotic mechanisms for ankle function evaluation, measurement and physiotherapy. For the choice, design and operation of the mechanism the kinematics of the foot is described. This is based on a kinematics model of foot adopted from biomechanics literature, under the hypothesis that foot kinematics is similar to that of a $2 R$ serial robot. A 3D scanner and an inertial sensor were used in order to fully specify the design framework by studying a larger sample of healthy subjects. Our experimental analysis confirms and enhances the $2 \mathrm{R}$ foot model, and leads us to the choice of the specific mechanism. We compute the required workspace and thus address the issues required for a complete and efficient design. We compare mechanisms based on serial and parallel robots, and choose a parallel tripod with an extra rotation axis for its simplicity, accuracy and generality. The robot must be capable to perform several multi-axis motions and sustain a significant range of forces and torques. The kinematic analysis of the robot confirms that it can follow all the range of foot movements.
\end{abstract}

\section{INTRODUCTION}

Many people have kinetic problems that are owed in ankle injuries since they are very common and so they often require rehabilitation. The equipment used for ankle rehabilitation is usually simple devices such as elastic bands (fig. 1), foam rollers and wobble boards [23]. These rehabilitation devices are typically used in regimens that include exercises both in the clinic and at home. They don't provide diagnostic and networking capabilities to allow therapists to remotely monitor the patient's progress. In addition, they are rarely interactive, making exercising monotonous. More importantly, they cannot be used for assessment, and execution of all rehabilitation tasks, hence the interest for an automatic device for complete physiotherapy and evaluation is big.

Several researchers proposed automatic mechanisms to help the physiotherapist [1]. Quite successful is the work at Rutgers University, including a haptic interface for ankle and hand rehabilitation. For ankle rehabilitation, a haptic interface was developed [5], based on a Stewart platform that applies variable forces and virtual reality exercises on the foot, including remote control operation. However, its 6DOF structure is redundant and there is no report for the design criteria; we aim at a smaller and more economic alternative, easier to move and operate. We also emphasise

\footnotetext{
Manuscript received July 5, 2008.

C.E. Syrseloudis is with the Department of Informatics and Telecommunications, National Kapodistrian University of Athens (NKUA), GREECE (phone: +302107275342; e-mail:c.sirseledi.uoa.gr)

I.Z. Emiris is with the Department of Informatics and Telecommunications, National Kapodistrian University of Athens (NKUA), GREECE (e-mail:emirisedi.uoa.gr)
}

to a device with adaptation to the specific characteristics of each patient's foot.

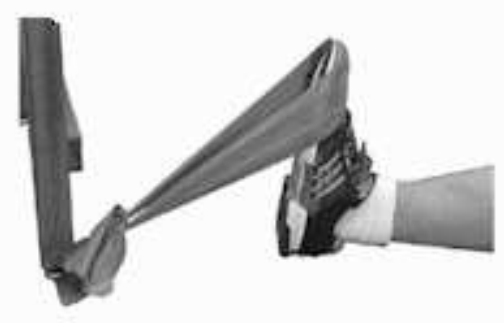

Fig. 1. Elastic-band rehabilitation exercise.

In [8], the study of ankle injuries and ankle functional anatomy was based on an orientation image space; 3 platform-type ankle rehabilitation mechanisms were considered and their mobility, stiffness and constraints analyzed. But other design criteria are not analyzed nor is the construction and functional operation of such platforms.

Parallel robot design concerns the geometric parameters calculation of a parallel robot with specific architecture satisfying several criteria [17]. A method for parallel robot design is based on interval analysis as it is proposed [12]. This involves multidesign criteria (workspace covering, accuracy bounds satisfaction, stiffness etc). A procedure with multiobjective optimization design was proposed in [6].

We focus on the design specifications of a simple robot for ankle injuries treatment. The allowable motions of foot are described and the foot trajectories during physiotherapy exercises are defined. Previous works were based on approximate estimations for the range of foot motions and they make no reference for the allowable movements. Here we use a foot kinematics model, based on the biomechanics literature. We show that not all movements of the ankle joint are allowable, since the rotation axes in the ankle have a specific position and the trajectories of the foot are constrained.

By using an existing kinematics model of the foot we compute the translation and orientation workspace of the robot. Extensive experiments with a 3D scanner and an inertial sensor have been performed to determine the kinematics behavior of the foot. The data obtained define the translation and orientation workspaces, and the velocity and acceleration ranges [22]. The manipulator must have the ability to follow the basic human foot motions in the 
plantarflexion-dorsiflexion and inversion- eversion directions with the upper leg fixed and sustain a significant range of forces and torques. We compare serial and parallel robot architectures and choose a parallel tripod with an extra rotation axis for its modularity, simplicity, accuracy and generality.

\section{AnKLe Related Foot Kinematics}

The human ankle has a complex multi-joint structure. The central bone is the talus. Its surrounding bones are the calcaneus, the navicular and the cuboid; they are responsible for the rotation of the ankle joint in 3D. The upper part of the talus articulates with the shank segment through the tibia and fibula bones. This is the upper ankle joint (UAJ); it supports the rotational dorsiflexion/plantarflexion motion.

The lower talus articulates with the calcaneus at the talocalcaneal joint and with the navicular at the talonavicular joint. The navicular also articulates with the cuboid and the cuboid with the calcaneus. The movements between the fore bones are strictly coupled. Motion of the foot wrt the talus is regarded as a rotation about the (fixed) subtalar joint (STJ); this supports the rotation supination/pronation, which has an inversion/eversion and an abduction/adduction component.

We adopt an ankle kinematics model from biomechanics literature [10]. The lower limb is assumed to be composed of 3 rigid links capable to rotate between each other: the shank, the talus and the foot configuring a serial manipulator with 2 rotation joints. These joints are the UAJ and STJ and support the main rotations of the foot: plantarflexion/dorsiflexion, inversion/eversion. The size of foot bones and their relative positions as well as the orientation of rotation axes determine the foot kinematics. Many factors influence the joint rotation, e.g. shape of articular surfaces, position of rotation axes. Constraint and resistance on the foot motions are due to ligaments, capsules and tendons.

The parameters of this model are specified by a number of point markers that have been assigned on the human foot as in fig. 2. These point markers are used to obtain a set of distance measurements. We assign frame $O_{1}$ at the knee, centered between $P_{1}, P_{2}$, with the $z$-axis parallel to $\left(P_{1}, P_{2}\right)$ and the $x$-axis vertical, passing through the midpoint of $\left(P_{3}, P_{4}\right)$ (fig. 3). By using the Denavit-Hartenberg(D-H) method we assign relative frames $O_{i}$ between the moving links. $T_{i}^{i+1}$ is the transformation matrix from $O_{i}^{i+1}$ into $O_{i}$.

The transformation matrix from the last into the first coordinate system is given from the relationship $T_{1}^{4}=T_{1}^{2} T_{2}^{3} T_{3}^{4}$, where $O_{4}$ is placed on the $P_{8}$. For a point $P=\left[\begin{array}{llll}x & y & z & 1\end{array}\right]^{T}$ on the last(foot) coordinate system the above transformation into the first(shank) coordinate system can be expressed as $P_{o}=\left[\begin{array}{llll}x_{o} & y_{o} & z_{o} & 1\end{array}\right]^{T}$ :

$$
P_{o}=T_{1}^{4} P
$$

from which the coordinates $x_{o}, y_{o}, z_{o}$ are nonlinear functions $f_{i}\left(a_{i}, \alpha_{i}, d_{i}, \vartheta_{i}, x, y, z\right)$ of the D-H parameters $a_{i}, \alpha_{i}, d_{i}, \vartheta_{i}$. These equations give a parametric formula in the movement of $P$ wrt the fixed coordinate system of the shank. The independent variables of the model are the angles

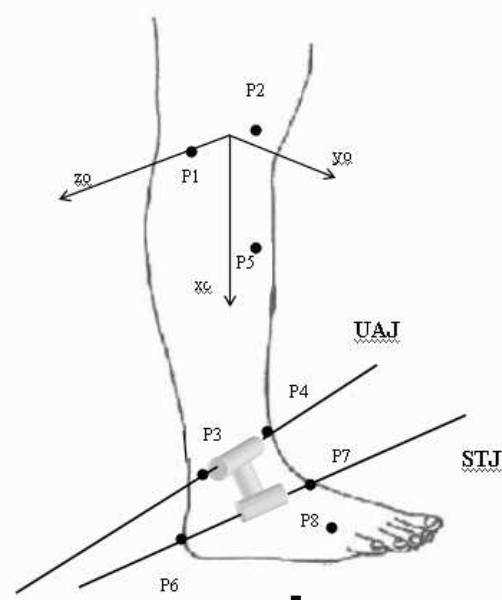

Fig. 2. Point markers on the foot and ankle's main rotation axes.

$\vartheta_{2}$ (dorsiflexion/plantarflexion) and $\vartheta_{3}$ (inversion/eversion) while $\vartheta_{1}$ is constant. According to the right-hand coordinate system assigned to the lower limb, the signs for rotation angles are: dorsiflexion(+), plantarflexion(-), eversion(+) and inversion $(-)$. Movements of the left leg are assumed to be the mirror-image of the right leg [10]. The parameters $\alpha_{i}, a_{i}, d_{i}$ depend on the foot anatomy and size.

In [10] the transformation matrices were estimated for a male subject. Standard instruments were used to measure the distances between the bony landmarks. After the calculation of several internal distances using the triangulation technique, the redundant distance method was used for the calculation of the transformation matrices between the foot to talus and between the talus to shank frames. From these data, a kinematics model of the foot was produced that is based on homogeneous matrix transformations expressed in Euler angles.

Here we were based on the given homogeneous transformation matrices of [10]. We calculated the relative orientation of axes of the lower limb, the vertical distances between them as well as the offsets between the vertical distances. In this manner we obtained the D-H parameters of the foot.

We take $\vartheta_{d p}, \vartheta_{i e}$ as the new variables for the dorsiflexion/plantarflexion and inversion/eversion angles measured from the standing posture and insert $\vartheta_{2}=\vartheta_{2}^{o}+\vartheta_{d p}, \vartheta_{3}=$ $\vartheta_{3}^{o}+\vartheta_{i e}$ into eqts (1). Now $\vartheta_{d p}, \vartheta_{i e}$ are the input variables of the model. Common ranges for the movements are $-40^{\circ} \leq$ $\vartheta_{d p} \leq 30^{\circ},-20^{\circ} \leq \vartheta_{i e} \leq 20^{\circ}$ [19].

Based on the model, we specify the workspace produced by the foot when the inputs take values through all the range of motions. Our first requirement is the shank to be fixed and vertical wrt the World Coordinate system attached to the base of the robot.

\section{A. Translation workspace}

We fix a point $P_{f}$ on the sole of the foot under the ankle where the center of the robot's end-effector will be attached. We assume $P_{f}$ is on the positive axis of the knee's frame and has a distance equal to this of $P_{6}$. The workspace produced by 

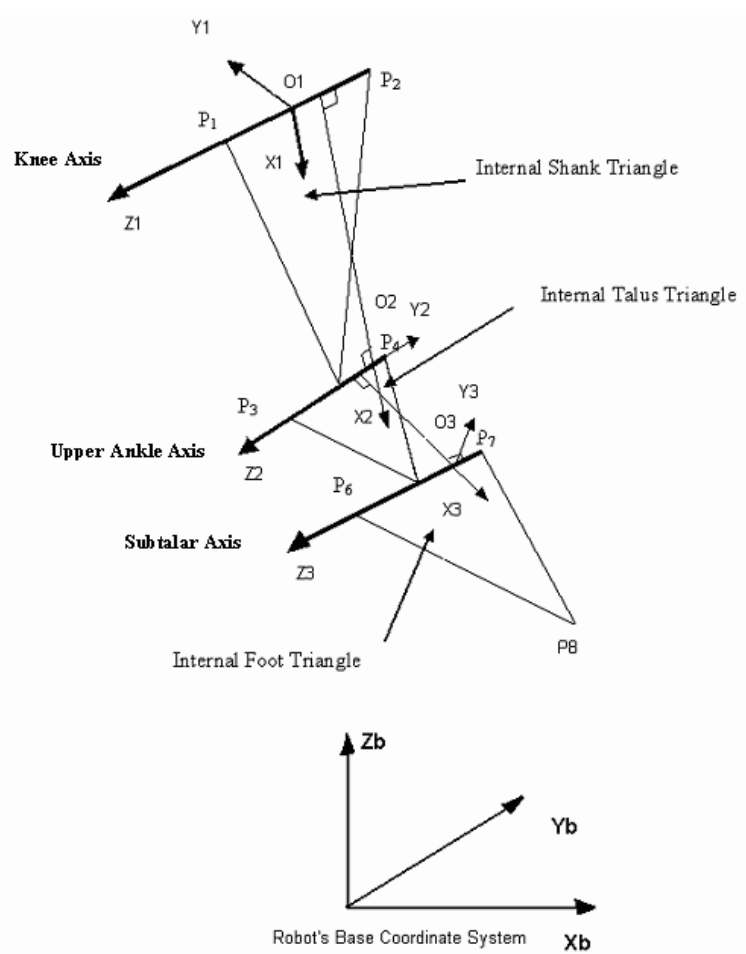

Fig. 3. Coordinate frames assignment on the foot and the internal triangles.

the foot will be derived from the motion study of $P_{f}$. By eqts (1) and letting inputs $\vartheta_{d p}, \vartheta_{i e}$ run through their entire regions, $P_{f}$ traces the surface of fig. 4(a). Assuming the right and left foot motions are mirror-images, the left foot workspace is a mirror-image surface of fig. 4(a). Feet of every size and anatomy produce a surface as in fig. 4(a). The geometric characteristics of this surface (e.g. shape, curvature), depend on $\alpha_{i}, a_{i}, d_{i}$. Every trajectory traced by $P_{f}$ is within this surface.

TABLE I

COORDINATE RANGES OF A POINT ON THE SOLE UNDER THE ANKLE.

\begin{tabular}{|l|l|l|l|l|l|}
\hline \multicolumn{2}{|c|}{$\Delta \mathrm{X}=56 \mathrm{~mm}$} & \multicolumn{2}{c|}{$\Delta \mathrm{Y}=41.7 \mathrm{~mm}$} & \multicolumn{2}{c|}{$\Delta \mathrm{Z}=17.3 \mathrm{~mm}$} \\
\hline Min $\mathrm{X}$ & Max X & Min Y & Max Y & Min Z & Max Z \\
\hline-33.5 & 22.4 & -19.6 & 22 & -2.8 & 14.4 \\
\hline
\end{tabular}

\section{B. Orientation workspace}

We compute the orientation of the foot when its axes are rotated in specific angles. First, we establish a reference frame with its origin at $P_{f}$. The axes are parallel with those of the base frame when the foot is in the neutral position. The rotation angles $\operatorname{roll}(\alpha), \operatorname{pitch}(\beta), \operatorname{yaw}(\gamma)$ of this frame wrt the base frame are the rotation angles of the sole of the foot as well as of the robot's end-effector that performs the task. The given foot model, when the inputs $\vartheta_{d p}, \vartheta_{i e}$ take

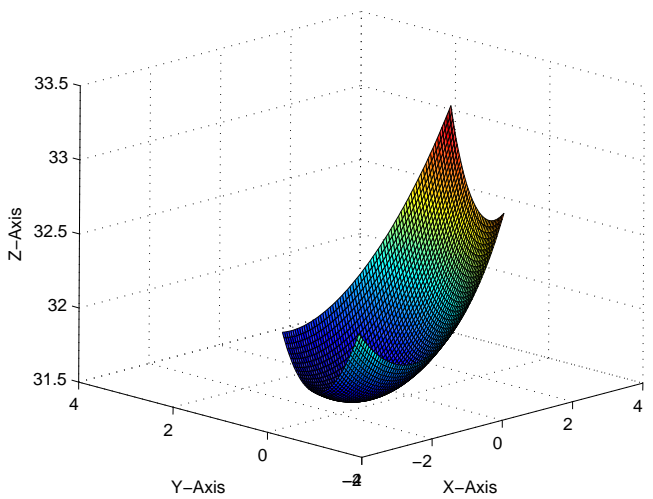

(a) Translation

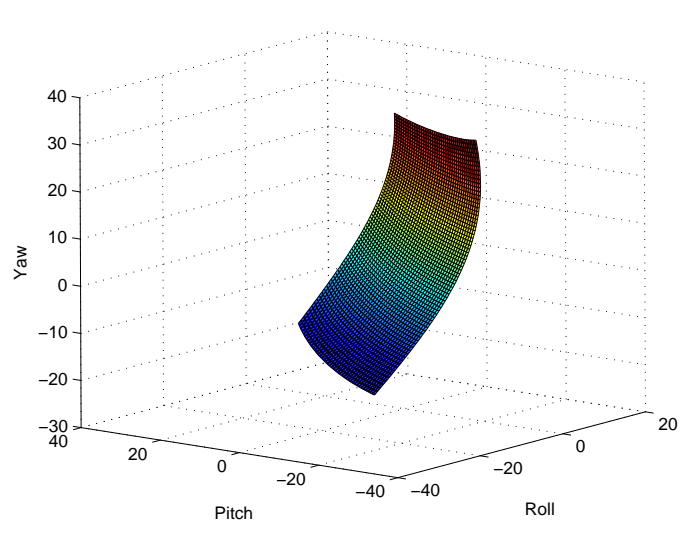

(b) Rotation

Fig. 4. Workspace from a point on the sole under the ankle.

all values in their ranges, give the orientation workspace of fig. 4(b).

TABLE II

ORIENTATION RANGES BASED ON THE MODEL.

\begin{tabular}{|c|l|l|l|l|l|}
\hline \multicolumn{2}{|c|}{$\Delta \alpha=30.56 \mathrm{deg}$} & \multicolumn{2}{|c|}{$\Delta \beta=76.58 \mathrm{deg}$} & \multicolumn{2}{c|}{$\Delta \gamma=62.49 \mathrm{deg}$} \\
\hline $\operatorname{Min} \alpha$ & $\operatorname{Max} \alpha$ & $\operatorname{Min} \beta$ & $\operatorname{Max} \beta$ & $\operatorname{Min} \gamma$ & $\operatorname{Max} \gamma$ \\
\hline-20.71 & 9.84 & -39.95 & 36.63 & -25.34 & 37.15 \\
\hline
\end{tabular}

By assuming that the angle axes parameters in eqts (1) are found in well specified intervals, we will specify the extended workspace produced by the model. In this way will include many feet with ankle axes in different angles. In [13] the orientation of the lower limp rotation axes and the ranges in the relevant angles between them were measured. The results depend on the position of the foot even for a given patient. Different patients will give different results. We conclude that the model parameters are quite uncertain and so the model must be extended to include uncertainties. Taking into account the orientation ranges of ankle axes from [13] and by Maximize, Minimize optimization functions of Maple, eqts (1) yield table III. 
TABLE III

EXTENDED COORDINATE RANGES OF A POINT ON THE SOLE UNDER THE ANKLE.

\begin{tabular}{|l|l|l|l|l|l|}
\hline \multicolumn{2}{|c|}{$\Delta \mathrm{X}=114.2 \mathrm{~mm}$} & \multicolumn{2}{c|}{$\Delta \mathrm{Y}=98.9 \mathrm{~mm}$} & \multicolumn{2}{c|}{$\Delta \mathrm{Z}=37.3 \mathrm{~mm}$} \\
\hline Min X & Max X & Min Y & Max Y & Min Z & Max Z \\
\hline-67.6 & 46.9 & -45.5 & 53.4 & -7.6 & 29.7 \\
\hline
\end{tabular}

\section{Experimental data}

A Mephisto 3D Scanner [2] was used to take images of the right foot sole in 11 adult healthy subjects of different age (22-42 years old), height, weight and gender. We used 5 positions: Neutral, max. Dorsiflexion, max. Plantarflexion, max. Supination, max. Pronation. The reference is a central point on the sole under the ankle because this point will be controlled by the robotic device. The experimental data are gave us the maximum volume of $\Delta \mathrm{X}=103.5 \mathrm{~mm}, \Delta \mathrm{Y}=98.3$ $\mathrm{mm}, \Delta \mathrm{Z}=74.9 \mathrm{~mm}$.

To measure orientation angles, rotation velocities, and accelerations, we performed experiments with the MTi motion sensor of XSens Motion Technologies [26]. This sensor provides and records pitch, roll and yaw angles, rate of turn and linear accelerations in axes $X, Y, Z$. We used the right foot of 5 adult healthy humans of both genders and different heights. Their shank kept vertical and fixed and the only moving part was the foot. The sensor was attached on the foot sole under the ankle. Data were recorded during dorsiflexion-plantarflexion and inversion-eversion throughout the entire range of movement. Fig. 5 shows roll, pitch and yaw wrt time of a foot in extreme rotational movement. Fig. 7 shows angular velocities wrt time in extreme rotational movement. The $\min / \max$ values are shown in tables IV (roll, pitch and yaw), V (linear accelerations) and VI (angular velocities).

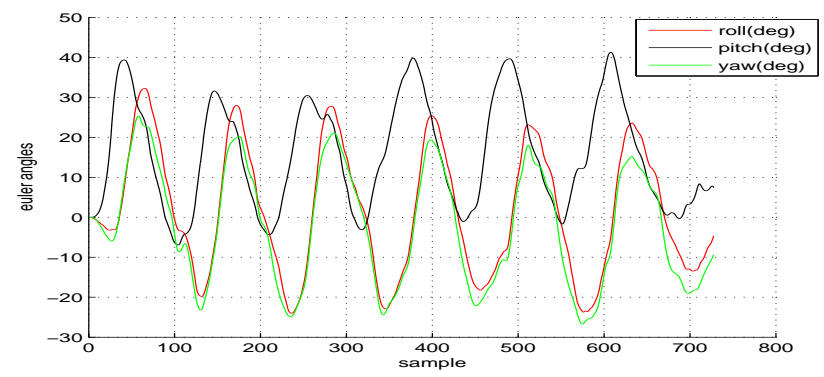

Fig. 5. Euler angles of foot on the extreme rotational motion.

TABLE IV

ORIENTATION RANGES BASED ON OUR EXPERIMENTS.

\begin{tabular}{|c|l|l|l|l|l|}
\hline \multicolumn{2}{|c|}{$\Delta \alpha=112.31 \mathrm{deg}$} & \multicolumn{2}{|c|}{$\Delta \beta=117.2 \mathrm{deg}$} & \multicolumn{2}{c|}{$\Delta \gamma=104.68 \mathrm{deg}$} \\
\hline $\operatorname{Min} \alpha$ & $\operatorname{Max} \alpha$ & $\operatorname{Min} \beta$ & $\operatorname{Max} \beta$ & $\operatorname{Min} \gamma$ & $\operatorname{Max} \gamma$ \\
\hline-45.79 & 66.52 & -50.63 & 66.57 & -40 & 64.68 \\
\hline
\end{tabular}

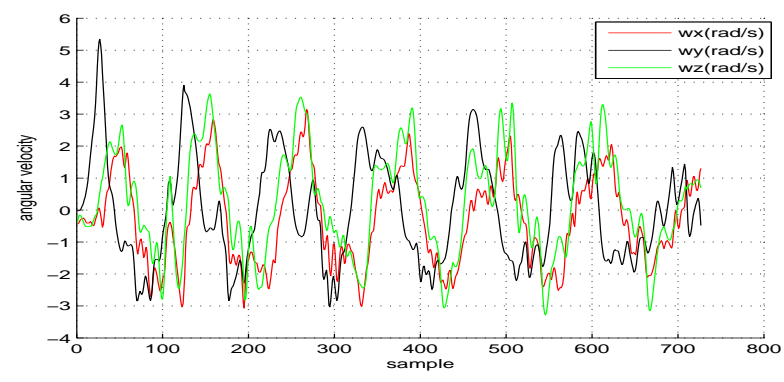

Fig. 6. Angular velocities of foot in extreme rotational motion.

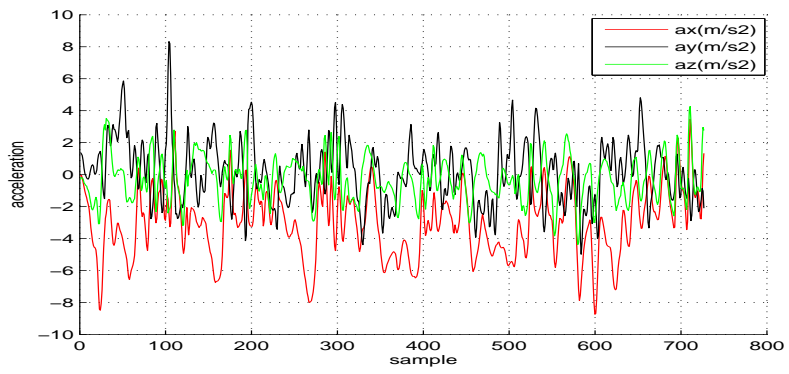

Fig. 7. Linear acceleration of foot in extreme rotational motion.

Previous measurements of ankle muscle strength have shown that the torque producing potential of the plantraflexors is smaller than $200 \mathrm{Nm}$ [21],[16]. For the dorsiflexors and the ankle invertors and evertors the maximum torque values produced during a strength test are smaller than $100 \mathrm{Nm}$ [16]. Thus, our platform will operate up to $200 \mathrm{Nm}$ [22], to handle torque-producing tasks at different velocities during concentric or eccentric muscle actions.

We got useful information when we consulted the gait analysis literature [7]. Gait analysis studies the lower limb behavior during walking, running etc. Also, the appropriate use of musclulo-skeletal simulation software [3] can help us to better understand the influence of the reaction forces on the lower limbs.

\section{ROBOT-BASED PHYSIOTHERAPY MECHANISMS}

For the selection of the robotic device we considered existing and on-going research on robot physiotherapy [4],[20]. We had to select between several candidate mechanisms. Wire robots are flexible, light and easier to construct but they are not stiff nor stable enough for our purpose. An orthosis is a device applied externally to a part of the body [24]. It is used to correct deformity, improve function or relieve symptoms of a disease by supporting or assisting the musculo-neuro-skeletal system; however, to design and develop a robotic orthosis is heavy for the patient, costly and difficult to construct.

In general, industrial serial robots provide the necessary accuracy, programming efficiency and capabilities to perform the task [25],[20]. On the other hand, they are redundant and expensive for the current approach. One candidate was a 3axes serial robot in which the rotation is internal so that the 
TABLE V

LINEAR ACCELERATION RANGES OF THE FOOT.

\begin{tabular}{|l|l|l|l|l|l|}
\hline \multicolumn{2}{|c|}{$\Delta \alpha_{x}=30.56 \mathrm{~m} / \mathrm{s}^{2}$} & \multicolumn{2}{c|}{$\Delta \alpha_{y}=76.58 \mathrm{~m} / \mathrm{s}^{2}$} & \multicolumn{2}{c|}{$\Delta \alpha_{z}=62.49 \mathrm{~m} / \mathrm{s}^{2}$} \\
\hline $\operatorname{Min} \alpha_{x}$ & $\operatorname{Max} \alpha_{x}$ & $\operatorname{Min} \alpha_{y}$ & $\operatorname{Max} \alpha_{y}$ & $\operatorname{Min} \alpha_{z}$ & $\operatorname{Max} \alpha_{z}$ \\
\hline-14.88 & 18.68 & -21.85 & 28.94 & -10.66 & 19.37 \\
\hline
\end{tabular}

TABLE VI

ANGULAR VELOCITIES RANGES OF THE FOOT.

\begin{tabular}{|l|l|l|l|l|l|}
\hline \multicolumn{2}{|c|}{$\Delta \omega_{x}=20.63 \mathrm{rad} / \mathrm{sec}$} & \multicolumn{2}{c|}{$\Delta \omega_{y}=16.45 \mathrm{rad} / \mathrm{sec}$} & \multicolumn{2}{c|}{$\Delta \omega_{z}=13.36 \mathrm{rad} / \mathrm{sec}$} \\
\hline Min $\omega_{x}$ & Max $\omega_{x}$ & Min $\omega_{y}$ & Max $\omega_{y}$ & Min $\omega_{z}$ & Max $\omega_{z}$ \\
\hline-9.3 & 11.33 & -8.43 & 8.02 & -7.64 & 5.72 \\
\hline
\end{tabular}

axes can be adapted to be collinear to the rotational axes of the ankle. This is too restrictive and overall, it does not offer a high stiffness, rigidity and manipulability.

\section{A. Parallel mechanisms.}

Parallel robots [18] are closer to our application because our workspace is confined and the robot mass to force handling ratio is much smaller. The forces that can be exerted to the robot when stepping on the device are quite high, however the torque for performing the physiotherapy exercises should be much smaller and accurately controlled. Therefore we decompose the forces and torques exerted on the ankle to distinguish between conditions which are acceptable according to the training session against others that can harm the ankle.

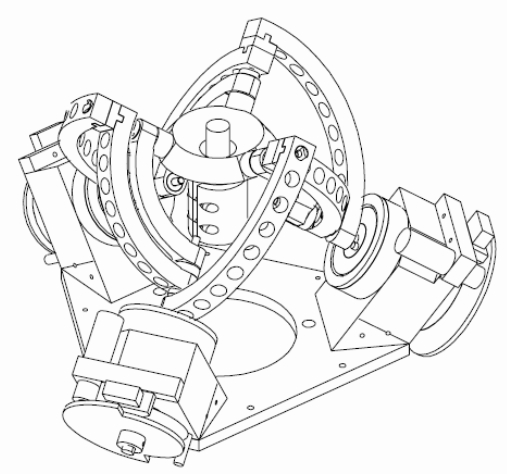

Fig. 8. The Agile-eye 3DOF parallel robot [11].

One possible solution should be the Agile-Eye 3-DOF platform [11]. Its main feature is that the moving platform rotates spherically around an external point and it has large orientation workspace. This should match in our case if the foot movements were based on old fashioned spherical joint ankle models.

\section{B. A Tripod-Based Parallel Mechanism}

The most promising candidate is a properly sized tripod (Fig. 9) together with an additional rotational axis, which provides all necessary characteristics and flexibility. This is

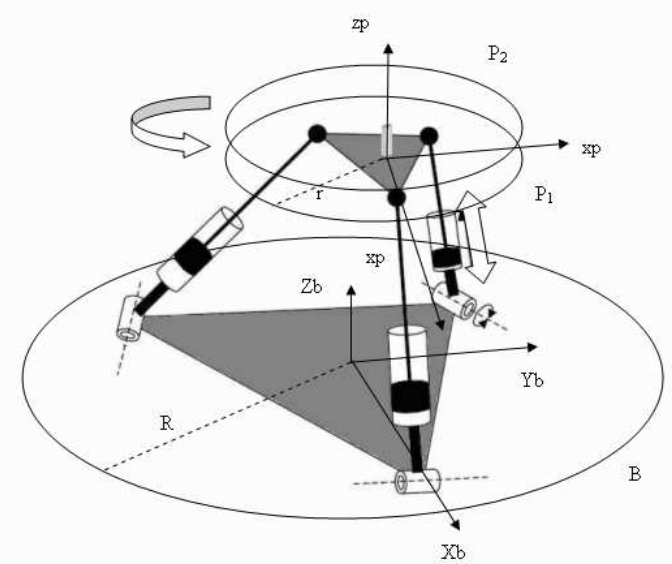

Fig. 9. The Tripod based parallel robot.

much smaller and simpler than a Stewart platform and has no redundancy.

The kinematics of this mechanism has been studied from Lee and Shah in [14]. It has two rotational (pitch, roll) and one translational $(\mathrm{z})$ degrees of freedom. Rotation about $\mathrm{z}$ axis is not allowed. As we have shown in the foot kinematics section the amount of yaw angle of the foot is significant. For this reason we have added an extra rotation axis on the moving platform $P_{1}$ witch gives rotation capabilities to the platform $P_{2}$ wrt $P_{1}$.

The inverse kinematics is related to the calculation of the leg lengths $l_{i}$ when the orientation and translation of the moving platform are given. If we denote by $\alpha$ and $\phi$ the two independent orientation angles the orientation matrix $\mathrm{R}$ is given from

$$
R=\left[\begin{array}{ccc}
k_{x}^{2}+k_{y}^{2} C \phi & k_{x} k_{y}(1-C \phi) & k_{y} S \phi \\
k_{x} k_{y}(1-C \phi) & k_{y}^{2}+k_{x}^{2} C \phi & -k_{x} S \phi \\
-k_{y} S \phi & k_{x} S \phi & C \phi
\end{array}\right]
$$

where $C \phi=\cos (\phi), S \phi=\sin (\phi), C \alpha=\cos (\alpha), S \alpha=$ $\sin (\alpha), k_{x}=C \alpha, k_{y}=S \alpha$. If $O_{p}=[x c, y c, z c]^{T}$ is the center of the moving platform wrt the base frame and $A_{i}=\left[x_{A i}, y_{A i}, y_{A i}\right]$ the spherical joints coordinates wrt the moving platform frame then the leg lengths are calculated from

$$
l_{i}^{2}=\left\|R * A i-B i+O_{p}\right\|
$$

For given angles $\alpha, \phi$ the dependent variables xc, yc of the center of the moving platform wrt the base frame are given from

$$
\begin{gathered}
x c=\frac{1}{2} r(1-C \phi) C_{2 \alpha} \\
y c=-\frac{1}{2} r(1-C \phi) S_{2 \alpha}
\end{gathered}
$$

After properly selection of $\alpha, \phi$ and $\mathrm{r}$ we can define $\mathrm{xc}$, yc from eqs. 4,5 . These must be equal to the $\mathrm{x}, \mathrm{y}$ coordinates 
of $P_{f}$ expressed in the base frame. Placement of the robot in $\mathrm{z}$ axis is arbitrary [14]. In this way, the tripod with the an extra rotation axis on the moving platform can trace feet movements.

The design of the robot concerns the geometric parameters calculation of the robot so that the moving platform to be found within the given workspace. Also it must achieve accelerations, velocities and forces inside the regions that have been defined in the previous sections. Additional design criteria are:

- Singularities: the platform must avoid singular configurations within the given workspace.

- Accuracy of the platform: it is the transmission of actuators errors to the platform. In our case error of $1 \mathrm{~mm}$ in translation and $1^{\circ}$ in orientation are acceptable.

- Stiffness of the platform: the platform must be quite stiff because it handles significant amount of foot forces.

\section{CONCLUSION AND Future WORKS}

In this paper several topics related with the functional evaluation and rehabilitation of human ankle via a robotic device have been discussed. At first, a kinematic model of the foot is adopted from the biomechanics literature in order to describe the foot motions and the desired workspace calculation of such a robot. We look in detail to the ankle structure as well as in the ankle axes position e.g the axis of rotation inversion/eversion is not parallel to the horizontal plane. Experiments and quantitative analysis utilizing a 3D scanner and an inertial and orientation sensor provided the necessary data to enhance the design features of the robotic device.

This workspace and trajectory characteristics will be used for the parametric design of the geometric parameters of the aforementioned parallel tripod with an additional rotation axis, chosen for its modularity, rigidity and simplicity.

The interesting subject of foot's D-H parameters identification through the robot movements is now studying. This is crucial since the device will be used from different patients with different anatomical parameters [13]. From the expansion of equations 1 are produced the following functions $P_{f i}=f_{i}\left(a_{i}, \alpha_{i}, d_{i}, \vartheta_{d p i}, \vartheta_{i e i}, x, y, z\right)$. These leads to a system of nonlinear equations that contains the $\mathrm{x}, \mathrm{y}, \mathrm{z}$ coordinates of $P_{f}$. By implementing algebraic elimination techniques via resultants [9] and by use of Multires package of Maple we eliminate the non measurable angles $\vartheta_{d p i}, \vartheta_{i e i}$. By implementing Non Linear Least Squares optimization on the eliminated final system we identify the structural parameters of the foot. The problem is quite difficult since it produced a large size matrix with many parameters. So the final eliminated system is hard to compute. We try to overcome this by implementing efficient algorithms (interpolation, parameter reduction etc.) to reduce the complexity of the calculations.

\section{ACKNOWLEDGMENT}

We thank Dr. J-P. Merlet and Dr. D. Daney of INRIA, Dr. C.N. Maganaris of IRM Manchester Metropolitan University and C. Konaxis of NKUA for their useful advice.

\section{REFERENCES}

[1] http://laimuz.unizar.es/teledoc/home.html.

[2] http://www.thinglab.co.uk

[3] ANYBODY. http://www.anybodytech.com.

[4] M. Armada. www.iai.csic.es/iarp/sapr $/ 12_{\text {s }}$ pain $_{i} \operatorname{arp}_{j} c f_{2} 003$.pdf. International Advanced Robotics Programme (IARP) 22nd JOINT COORDINATING FORUM, pages 347-352, 2003.

[5] G. Burdea, M. Girone, M. Bouzit, V. Popescu, and J.E Deutsch. A Stewart platform-based system for ankle telerehabilitation. $A u$ tonomous Robots, 10:203-212, 2001.

[6] M. Ceccarelli, G. Carbone, and E. Ottaviano. An optimization problem approach for designing both serial and parellel manipulators. Proc. of MUSME 2005, Int. Symp. on Multibody Systems and Mechatronics, (3):pp 18-32, 2005. Uberlandia-Brazil.

[7] ClinicalGaitAnalysis. http://www.univie.ac.at/cga/

[8] J.S. Dai, T. Zhao, and C. Nester. Sprained ankle physiotherapy based mechanism synthesis and stiffness analysis of a robotic rehabilitation device. Autonomous Robots, 16:207-218, 2004.

[9] D.Daney and I.Z Emiris. Robust parellel robot calibration with partial information. Proc. IEEE Int. Conf. Robotics and Automation, pages 3262-3267, 2001. Seoul, S. Korea.

[10] J. Dul and G.E Johnson. A kinematic model of the human ankle. $J$. of Biomedical Engineering, 7:137-143, 1985.

[11] C.M. Gosselin and J. Wang. Singularity Loci of a Special Class of Sphericsl Three-degree-of-freedom Parallel Mechanisms with Revolute Actuators. Int. J. of Robotics Research, 21(7) pp.649-659, 2002.

[12] F. Hao and J-P. Merlet. Multi-criteria optimal design of parallel manipulators based on interval analysis. J. Mechanism and Machine Theory, 40(2):pp.157-171, 2005.

[13] R.E. Isman and V.T Inman. Anthropometric studies of the human foot and ankle. Bulletin of Prosthetic Research, 10-11:97-129, 1969.

[14] K. Lee, D.K. Shah. Kinematic Analysis of a three degrees of freedom in parallel actuated manipulator. IEEE J. of Rob. and Automation, Vol 4(3) pages 354-60, 2003.

[15] L.F Lee and V.N Krovi. Musculoskeletal simulation based optimization of rehabilitation program. Virtual Rehabilitation, pages 36-41, 2006.

[16] C.N. Maganaris, V. Baltzopoulos, D. Ball, and A.J. Sargeant. In vivo specific tension of human skeletal muscle. J. Appl. Physiol., 90(3):865-72, 2001.

[17] J-P. Merlet. Designing a parallel robot for a specific workspace. Technical Report 2527, 1995. INRIA.

[18] J-P. Merlet. Parallel Robots. Springer-Verlag, Second edition, 2006.

[19] B.M Nigg, V.Fisher, T.L. Allinger, J.R. Ronsky, and J.R. Engsberg. Range of motion of the foot as a function of age. Foot and Ankle, 16(6):336-343, 1992.

[20] J. Savage M. Levesley R. Richardson A. Cozens M.M Williams B. Bhakta P. Culmer, A. Jackson. From single to dual robotic therapy: A review of the development process of iPAM. Biomed. Robotics and Biomechatronics, pages 347-352, 2003.

[21] D. Sale, J. Quinlan, E. Marsh, AJ. McComas, and AY. Belanger Influence of joint position on ankle plantarflexion in humans. J. Appl. Physiol., 52(6):1636-42, 1982.

[22] C. Syrseloudis I. Emiris C. Maganaris T. Lilas Design Framework for a Simple Robotic Ankle Evaluation and Rehabilitation Device. To appear in 30th Int. Conf. IEEE Engineering in Medicine and Biology Society, Canada, 20-24 Aug. 2008.

[23] PerformBetter. http://www.performbetter.com.

[24] RCAI. http://www.rcai.com.

[25] A. Toth and I. Ermolaev. Robots with patients. Engineer IT, pages 60-62, 2006.

[26] XSens http://www.xsens.com 